\title{
Index for Measuring the Quality of Complementary Feeding Practices in Rural India
}

\author{
Aashima Garg and Ravinder Chadha \\ Department of Food and Nutrition, Lady Irwin College, University of Delhi, \\ Sikandra Road, New Delhi I I0 00 I, India
}

\begin{abstract}
This community-based cross-sectional study was undertaken to develop a complementary feeding index (CFI) to assess the adequacy of complementary feeding (CF) practices and determine its association with growth of infants, aged 6-12 months, in rural Indian population. The study was conducted in six villages of Ghaziabad district, Uttar Pradesh, India. A structured interview schedule was used for eliciting information from 151 mothers of infants, aged 6-12 months, on CF practices. Data on CF practices were scored using the CFI developed. Measurements of weight and length were taken. Bivariate and multivariate analyses were done using the SPSS software (version 13). The results revealed that the CF practices were suboptimal in the sample. The mean \pm standard deviation (SD) CFI scores ranged from a low value of 7.09 \pm 3.21 in 6-8 months old infants to a comparatively-higher value of 9.69 \pm 2.94 in 9-12 months old infants. Using the CFI it could be identified that infants $(n=151)$ had poor dietary diversity, with only $31 \%$ and $18 \%$ of the infants reportedly being fed the recommended number of food-groups during 6-8 and 9-12 months respectively. The food-frequency scores of the CFI showed that cereals and diluted animal milk were the major foodgroups fed to the infants in this setting. Analysis of nutritional status revealed that $24.5 \%$ of the infants were stunted (length-for-age [LAZ] <-2SD), 25\% were underweight (weight-for-age [WAZ] <-2SD), and 17\% were wasted (weight-for-age [WLZ] $<-2 S D)$. Significant associations $(\mathrm{p}<0.05)$ were observed between the meal-frequency and the dietary diversity of the CFs of infants aged 6-8 months and 9-12 months and the WAZ and LAZ indices of their nutritional status. On multivariate analysis of factors affecting the LAZ, WAZ and WLZ scores, the CFI was significantly associated $(\mathrm{p}<0.05)$ with LAZ whereas maternal education and breastfeeding frequency were significantly $(\mathrm{p}<0.01)$ associated with WAZ and WLZ. Per-capita income, parity, and birth-order were the significant $(\mathrm{p}<0.05)$ determinants of the CFI. The CFI developed is an exploratory attempt to summarize and quantify the key CF practices into a composite index, which would reflect the CF practices holistically. This index can be used as an easy tool by programme planners for identifying, targeting, and monitoring the deficient CF practices and also advocating the importance of the CF at policy level.
\end{abstract}

Key words: Community-based studies; Complementary feeding index; Complementary feeding practices; Cross-sectional studies; Infant nutritional status; India

\section{INTRODUCTION}

The link between malnutrition and infant-feeding practices has been well-established (1-2). Incidence of malnutrition rises sharply during 6-8 months

Correspondence and reprint requests should be addressed to:

Ms Aashima Garg

Senior Research Fellow

Manu Smrity

KI-8 Kavinagar

Ghaziabad, UP 201002

India

Email: aashi07@yahoo.com

Fax: 0120-4371836 of age in most countries, which coincide with period of complementary feeding (CF), and deficits acquired at this age are difficult to compensate later in childhood (2-3). In India, only 55\% of infants receive semi-solid or solid foods, in addition to breastmilk, during 6-8 months of age, showing faulty CF practices to be a significant problem of public health (4).

Efforts to measure and quantify the CF practices and to assess the strength of their association with child's nutritional status have been very limited till date due to methodological problems. This is because the CF practices encompass a series of inter- 
related behaviours that must be considered simultaneously and are, therefore, difficult to summarize into one or a few variables that accurately reflect these practices (5-6).

In this context, a composite index for a global assessment of infant and young child feeding was conceived in developing countries (5-7). This index summarized the salient young child-feeding practices, using a limited number of variables collected during the Demographic and Health Survey (DHS) in Latin American countries. The aim of the present study was to assess the feasibility of developing a CFI by adapting the index created by Arimond and Ruel (7) and to determine its association with growth of infants, aged 6-12 months, in rural Indian population. The CFI was created for using it as a quick tool to get a composite score of main CF practices of infants and to identify the deficient CF practices for a population, which would be targeted in the improvement intervention of CF practices.

\section{MATERIALS AND METHODS}

Sample and sample size

A community-based cross-sectional investigation was carried out in rural Uttar Pradesh, India. The site of the study comprised six purposively-selected villages, such as Bhojpur, Gadana, Bhikanpur, Kanoja, Abupur, and Ravli, from three rural community development blocks under the integrated child developmental services of Ghaziabad district, Uttar Pradesh, India. All the infants, aged 6-12 months, from the six villages were enlisted with the help of birth-records and survey-registers of Anganwadi worker (AWW) at each Anganwadi centre (AWC) [Anganwadi means a courtyard play centre, a childcare centre, located within the village itself, over a population of 1,000. The AWC is the focal point for grassroots-level service-delivery of six key services, namely supplementary nutrition, non-formal preschool education, immunization, health check-up, referral services, and nutrition and health education, including growth monitoring and promotion. Each AWC is run by one Anganwadi worker (AWW) and one helper]. In total, 320 infants were enlisted from these six villages. Infants with any congenital problem were excluded from the sample. Fifty percent of the infants were randomly selected for the survey using random number tables. The non-response rate was 5.5\% in the sample. Thus, in total, 151 mother-infant pairs formed the sample for the survey.

\section{Data collection}

An interview schedule was used for collecting in- formation on sociodemographic profile using standard of living index (SLI) (8), maternal characteristics, breastfeeding, CF practices, and morbidity profile of infants. SLI is a composite index for measuring the socioeconomic status of the household. It is a summary household measure and is calculated by adding the scores for type of house, toilet facility, source of lighting, main fuel for cooking, source of drinking-water, separate room for cooking, ownership of house, ownership of agricultural land, ownership of irrigated land, ownership of livestock, and ownership of durable goods. The index is calculated by summing the scores, which have been developed by the International Institute for Population Sciences, Mumbai and has been used in the National Family Health Survey 2, India (8) for measuring the socioeconomic status of households in the national survey. Index scores range from 0 to 14 for a low SLI to 15-24 for a medium SLI and 25-66 for a high SLI.

Before administering the interview schedule, an informed consent was taken from all the subjects or family members. Data on the intake of complementary foods were collected using 24-hour recall and beyond also using a seven days' food-frequency questionnaire. Anthropometric measurements taken were weight and length of target infants. Weight of infants was taken using a digital weighing scale (5 g sensitivity, manufactured by Goltech Digital Scales Company) and length using infantometer $(0.1 \mathrm{~cm}$ sensitivity, manufactured by Global Product Corporation) by standard procedures (9).

\section{Standardization of dietary data}

For collecting dietary data, the common household measures of the community, such as bowls, spoons, and ladles of different sizes, were standardized using a measuring cylinder. These standardized measures were then used for collecting dietary data at the community level. The dietary data collected using 24-hour recall and also using a food-frequency questionnaire were standardized by converting household measures to the standard quantities of raw and cooked foods using a digital weighing scale with a sensitivity of $1 \mathrm{~g}$ (manufactured by CAS Weighing Scales Company, India), by conducting cooking sessions in the community itself. As CFs vary remarkably in texture and consistency, this exercise helped know the accurate consistency of CFs fed to the infants.

Variables of CFI

The CFI was constructed for infants aged 6-12 
months based on the current $\mathrm{CF}$ recommendations for this age-group (10). A score was assigned using six variables: continued breastfeeding; avoiding bottle-feeding; timely initiation of CF; dietary diversity which was measured by the total number of food-groups the infant consumed during the past 24 hours; meal-frequency (past 24 hours); and food-frequency which was a measure of frequency of food-groups consumed by the infant during the preceding seven days. The consumption of the following seven food-groups was considered: cereals and tubers; pulses; milk and milk-products; meat and egg; vitamin A-rich fruits and vegetables; other fruits and vegetables; and foods made with oil, fat, and butter.

\section{Scoring of CFI}

The scoring of the CFI was done by assigning a score of ' 0 ' for a potentially-harmful practice and a score of ' 2 ' for a positive practice. Those practices in between these two were given a score of ' 1 '. The practices have been considered positive or negative based on the CF guidelines of the World Health Organization (WHO) for breastfed children (10). For the food-frequency score, each food-group was scored individually, and the scores for each one were summed to derive the final food-frequency score. Table 1 gives the CFI constructed for infants aged 6-12 months. Thus, theoretically, the CFI score would vary from a minimum of three to a maximum of 23 and grouped into terciles to form three categories of CF practices: low, medium, and high (5).

\section{Statistical analysis}

Data were analyzed using the SPSS software (version 13). Data on weight and length were used for calculating the following three summary indices of nutritional status: weight-for-age, length-for-age and weight-for-length, expressed in standard deviation (SD) units (z-scores) as per the child growth standards of WHO (11) using the WHO Anthro software. Distribution of quantitative variables was summarized as means $\pm \mathrm{SD}$. Multivariate regression analysis was used for determining whether the factors affecting the nutritional status and CFI remained significant after controlling for the potentially-confounding variables. All the test-results were considered significant for a $\mathrm{p}$ value of $<0.05$.

\section{RESULTS}

\section{Sociodemographic profile}

Of the 151 mothers of infants interviewed, the ma- jority (62\%) belonged to joint families, with 30\%, $42 \%$, and $28 \%$ of the infants belonging to low, medium, and high socioeconomic status respectively as per the SLI score. None of the mothers in the sample reported being engaged in any formal occupation, apart from working as agricultural labourer during harvest. The large majority (78\%) of the mothers were young with the mean $\pm \mathrm{SD}$ age of $25.32 \pm 4.84$ years, and $63 \%$ of them were literate. The mean $\pm \mathrm{SD}$ age of the infants was $9.32 \pm 1.88$ months, with 51 and 100 infants aged 6-8 months and 9-12 months respectively.

\section{Assessment of nutritional status}

In the sample, $24.5 \%$ of the infants were stunted (LAZ <-2SD), with $25.1 \%$ being underweight (WAZ $<-2 \mathrm{SD}$ ) and $17.2 \%$ wasted (WLZ <-2SD). Analysis based on age-group showed almost a four-fold increase in the prevalence of underweight and stunting from 6-8 months to 9-12 months of age.

Reported rates of morbidity were high; during the preceding 15 days, $48 \%$ had experienced diarrhoea, $42 \%$ fever, $39 \%$ cough, and $12 \%$ cough with rapid breathing (acute respiratory tract infection).

\section{Complementary feeding practices}

The percentage of mothers who reported selected CF practices is presented in Table 2. Breastfeeding was continued beyond six months of age by most mothers of the infants. The mean $\pm \mathrm{SD}$ breastfeeding frequency as reported by the mothers during the preceding 24 hours of survey was slightly higher in the age-group of 6-8 months (5.0 2.4$)$ than the age-group of 9-12 months (4.4 \pm 2.3$)$. About half of the mothers used bottles with nipples for feeding milk and other liquids to their infants, which may attribute to the high rates of morbidity in the sample. Of the 151 mothers, only nine introduced semi-solid/solid foods, along with breastfeeding at six months of age. The median (IQ) age of introduction of semi-solid/solid foods in the diet of infants was seven months (3). In both the age-groups, about $60 \%$ of the mothers fed their infants the recommended number of meals (10) in the previous 24 hours, with the median (IQ) meal-frequency for the total sample being 3 (2).

The dietary diversity scores were very low in both the age-groups, with only $31 \%$ and $18 \%$ of the mothers in the 6-8 and 9-12 months old infants reported feeding $\geq 3$ and $\geq 4$ food-groups respectively to their infants in the preceding 24 hours. The mean \pm SD number of food-groups consumed by the infants in the previous 24 hours ranged from 


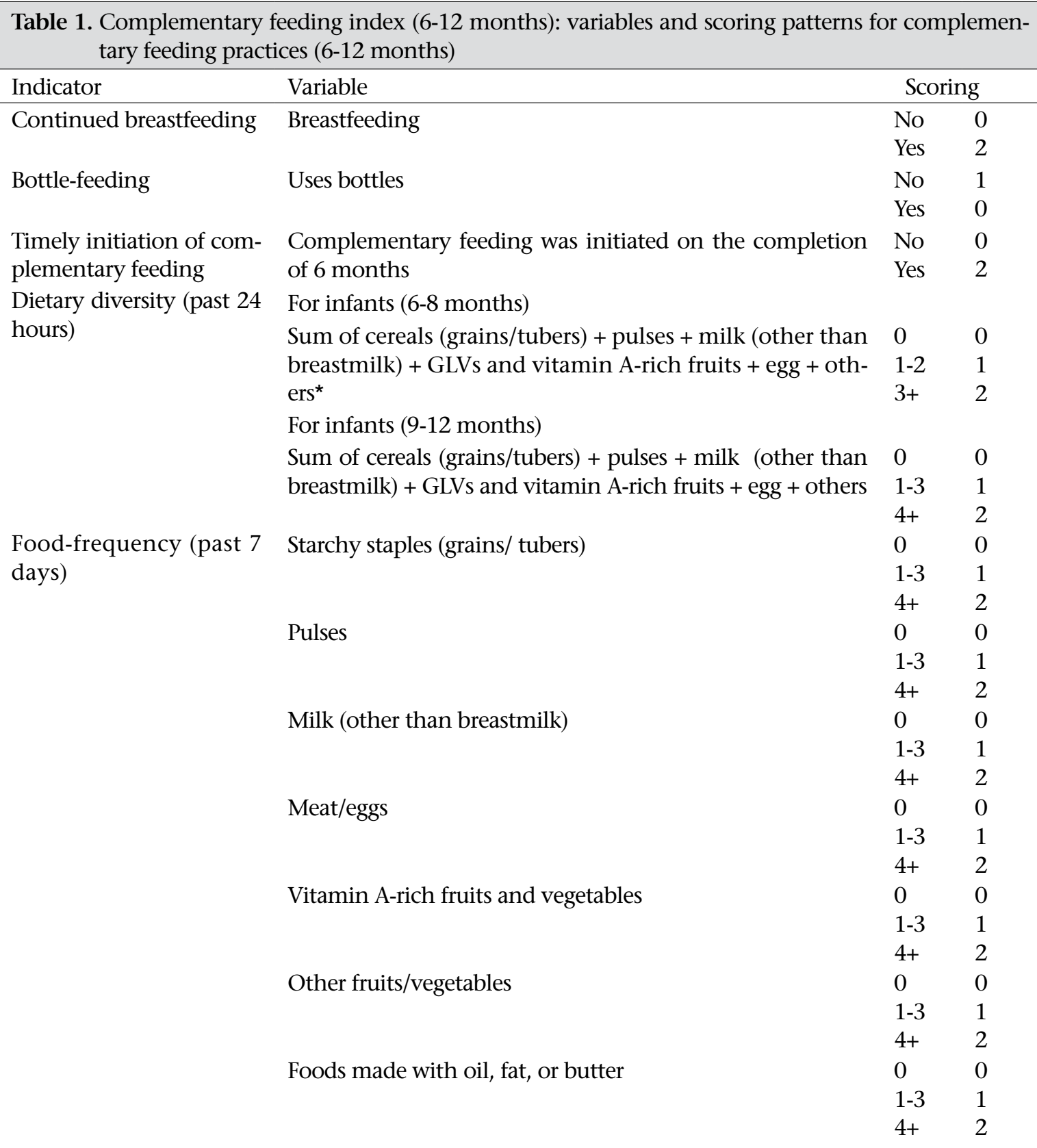

Meal-frequency (past 24

Food-frequency score $=$ sum of scores for starchy staples + pulses + milk + meat/egg + vitamin A-rich fruits/vegetables + foods made with fat hours)

For infants (6-8 months)

No. of times the child was fed in the past 24 hours

$\begin{array}{ll}0 & 0 \\ 1 & 1 \\ 2+ & 2\end{array}$

For infants (9-12 months)

No. of times the child was fed in the past 24 hours $\quad \begin{array}{lll}0 & 0\end{array}$

Minimum CFI score=3; Maximum CFI score=23; CFI score range: Low: $\leq 6$; Medium: 7-16; High: 17-23; *Other category includes fruits, other seasonal vegetables, fat, and sugar food-groups; CFI=Complementary feeding index; GLVs=Green-leafy vegetables 


\begin{tabular}{|c|c|c|c|c|}
\hline \multirow{2}{*}{ Feeding practice } & \multicolumn{2}{|c|}{ 6-8 months $(\mathrm{n}=51)$} & \multicolumn{2}{|c|}{ 9-12 months $(\mathrm{n}=100)$} \\
\hline & No. & $\%$ & No. & $\%$ \\
\hline Breastfeeding & 44 & 86.3 & 85 & 85.0 \\
\hline Use of baby-bottles & 28 & 54.9 & 54 & 54.0 \\
\hline Timely initiation of complementary food (at 6 months) & 3 & 5.9 & 6 & 6.0 \\
\hline \multicolumn{5}{|l|}{ Meal-frequency ${ }^{\star}$ (past 24 hours) } \\
\hline$\geq 2$ meals (for 6-8 months) & 31 & 60.8 & - & - \\
\hline$\geq 3$ meals (for 9-12 months) & - & - & 59 & 59.0 \\
\hline \multicolumn{5}{|l|}{ Dietary diversity $^{*}$ (past 24 hours) } \\
\hline$\geq 3$ food-groups (for $6-8$ months) & 16 & 31.4 & - & - \\
\hline$\geq 4$ food-groups (for 9-12 months) & - & - & 18 & 18.0 \\
\hline \multicolumn{5}{|l|}{ Food-frequency (gave at least once in the past 7 days) } \\
\hline Starchy staples & 22 & 43.1 & 80 & 80.0 \\
\hline Legumes & 15 & 29.4 & 46 & 46.0 \\
\hline Milk and milk-products & 30 & 58.8 & 76 & 76.0 \\
\hline Eggs & 4 & 7.8 & 17 & 17.0 \\
\hline Vitamin A-rich fruits/vegetables & 1 & 1.9 & 10 & 10.0 \\
\hline Other fruits/vegetables & 2 & 3.9 & 37 & 37.0 \\
\hline Foods made with oil/fat/butter & 2 & 3.9 & 37 & 37.0 \\
\hline
\end{tabular}

$1.72 \pm 1.3$ for $6-8$ months old infants to $2.58 \pm 1.06$ for 9-12 months old infants.

The individual combined food-frequency score for the past seven days ranged from 0 to 9 with the mean \pm SD score ranging from $2.39 \pm 1.99$ for $6-8$ months to $4.52 \pm 2.29$ for $9-12$ months old infants. Table 3 shows the individual mean food-frequency scores for various food-groups in both the agegroups. Cereals (wheat and rice) and animal milk were the major food-groups fed to infants of both the age-groups during the preceding week. In the former age-group, the mean frequency of feeding animal milk was the highest whereas, in the latter one, the frequency of feeding cereals was reported to be the highest as the usual foods being given to the infants included a piece of roti [traditional unleavened Indian bread] or biscuit with diluted animal milk. Despite all the vegetables being cooked in the households, they were not fed to the child, thinking he/she would not be able to digest it as the food was prepared by adding chilies and other spices. This also reflects in the mean food-frequency scores of vegetables and fruits for both the agegroups.

\section{Distribution of CFI}

The CFI scores varied from three to 17 in this sam- ple (for a theoretical maximum of 23). The CFI scores were divided into low (score $\leq 6)$, medium (score 7-16), and high (score 17 and 23), and in both the age-groups, the majority of infants $(56 \%$ and $86 \%$ in the age-groups of 6-8 and 9-12 months respectively) fell into the medium category of the CFI scores, with about $23 \%$ falling in the low category of the CFI scores, and only one infant in the age-group of 9-12 months having the high CFI score. The mean \pm SD CFI scores ranged from a low value of 7.09 \pm 3.21 in 6-8 months old infants to a comparatively-higher value of $9.69 \pm 2.94$ in $9-12$ months old infants, which is not even half of the maximum possible score of 23 , indicating a major gap in the CF practices. The CFI can be used for determining the gap between the practices being followed and the optimal practices and, hence, can be used as a screening tool at the community level.

\section{Associations with CFI}

Significant associations were observed between the nutritional status and the sociocultural factors, frequency of breastfeeding, dietary diversity, and meal frequency of infants. In the bivariate analysis, among the feeding practices, diversity in the diet of infants at 9-12 months of age had significant 


\begin{tabular}{|c|c|c|c|}
\hline \multirow[b]{2}{*}{ Food-group } & \multicolumn{3}{|c|}{ Mean \pm SD food-frequency score } \\
\hline & $\begin{array}{c}\text { 6-8 months } \\
(\mathrm{n}=51)\end{array}$ & $\begin{array}{c}9-12 \text { months } \\
(\mathrm{n}=100)\end{array}$ & $\begin{array}{c}\text { Total } \\
(\mathrm{n}=151)\end{array}$ \\
\hline Starchy staples & $0.66 \pm 0.8$ & $1.40 \pm 0.8$ & $1.15 \pm 0.9$ \\
\hline Legumes & $0.29 \pm 0.5$ & $0.51 \pm 0.6$ & $0.44 \pm 0.6$ \\
\hline Milk and milk-products & $1.14 \pm 0.9$ & $0.49 \pm 0.8$ & $1.37 \pm 0.9$ \\
\hline Eggs & $0.08 \pm 0.3$ & $0.17 \pm 0.4$ & $0.14 \pm 0.3$ \\
\hline Vitamin A-rich fruits/vegetables & $0.02 \pm 0.1$ & $0.10 \pm 0.3$ & $0.07 \pm 0.3$ \\
\hline Other fruits/vegetables & $0.16 \pm 0.4$ & $0.51 \pm 0.5$ & $0.33 \pm 0.5$ \\
\hline Foods made with oil/fat/butter & $0.04 \pm 0.2$ & $0.45 \pm 0.6$ & $0.31 \pm 0.6$ \\
\hline
\end{tabular}

associations $(\mathrm{p}<0.01)$ with all the three nutritional status indices. The meal frequency of CFs of infants was positively and significantly associated with weight-for-age and length-for-age indices both at 68 months $(\mathrm{p}<0.05)$ and 9-12 months $(\mathrm{p}<0.01)$.

Multivariate regression analysis was performed to determine the significance of correlations observed between the CFI and the nutritional status (weightfor-age and length-for-age) after controlling for the confounding variables, such as characteristics of child (age, gender, and birth-order), characteristics of mothers (age, education, and parity) and characteristics of households (family size, socioeconomic status, SLI, and per-capita income).

As depicted in Table 4, in the multivariate regression model, the CFI was observed to be significantly $(\mathrm{p}<0.05)$ associated with length-for-age but not with weight-for-age and weight-for-length indices. Among the characteristics of children, sex and age of infants significantly $(\mathrm{p}<0.05$ and $\mathrm{p}<0.01$ respectively) impacted their nutritional status. The age of mothers, educational status, and parity were the significant $(\mathrm{p}<0.05, \mathrm{p}<0.01$, and $\mathrm{p}<0.05$ respectively) determinants of the nutritional status of infants. The frequency of breastfeeding was also significantly associated with nutritional status.

Multivariate regression was also carried out to model the determinants of the CFI. All the maternal, child and household characteristics emerging as potential constraints in bivariate analysis were included in the regression model (Table 5). The results confirmed that parity and birth-order were the significant $(\mathrm{p}<0.05)$ determinants of CFI. The age of the child and per-capita income of the family also had a significant impact on the CFI scores of infants.

\section{DISCUSSION}

This study provides data on selected qualitative and semi-quantitative aspects of CF in a rural Indian setting, by creating a CFI by modifying and adapting a child-feeding index developed using data from the Demographic and Health Survey of five Latin American countries (5) and attempts to link them to three indices of nutritional status. A significant association was found between the CFI scores and the length-for-age index in this setting. There have been only a few attempts at creation of child-feeding index nationally (6) and internationally $(5,7,12)$.

The prevalence of underweight, stunting, and wasting was high in the sample, which may be attributed to the poor CF practices and high incidence of morbidity among the target infants. Consistent with the findings of the national data (8) and individual studies (13-14) in India, a 3- to 4-fold increase was observed in the prevalence of all the three nutritional status indices from 6-8 months to 9-12 months of age. Significant associations were observed between the socioeconomic status and the nutritional status, with the highest prevalence of stunting, wasting, and underweight in the low SLI group.

The CFI was significantly associated with SLI and per-capita income, indicating the poor CF practices in the low socioeconomic status group compared to the high socioeconomic status group, which is also consistent with the findings of other studies which reported better child-feeding practices in the high socioeconomic status group (5-6). The mean CFI scores were lower for 6-8 months vis-à-vis 9-12 months age-group. The lower scores in 6-8 months old infants indicate more gaps in the CF practices 


\begin{tabular}{|c|c|c|c|c|c|c|}
\hline \multirow{3}{*}{ Variable } & \multicolumn{6}{|c|}{ Nutritional status } \\
\hline & \multicolumn{2}{|c|}{ LAZ } & \multicolumn{2}{|c|}{ WAZ } & \multicolumn{2}{|c|}{ WLZ } \\
\hline & Coefficient & $t$-test & Coefficient & $t$-test & Coefficient & $t$-test \\
\hline Complementary feeding index & $0.18^{\$}$ & $2.04^{*}$ & 0.14 & 1.55 & 0.03 & 0.31 \\
\hline Breastfeeding-frequency & 0.09 & 1.24 & $0.17 \$$ & $2.32^{*}$ & $0.17 \$$ & $2.14^{*}$ \\
\hline \multicolumn{7}{|l|}{ Characteristics of children } \\
\hline Sex & $0.16^{\$}$ & $2.12^{*}$ & 0.07 & 0.91 & 0.05 & 0.59 \\
\hline Age (months) & $-0.34 \$$ & $-4.24^{\star \star \star}$ & $-0.23 \$$ & $-2.86^{\star *}$ & -0.08 & -0.98 \\
\hline Birth-order & -0.20 & -0.42 & 0.34 & 0.72 & 0.71 & 1.43 \\
\hline \multicolumn{7}{|l|}{ Characteristics of mothers } \\
\hline Age & -0.01 & -0.10 & 0.20 & 1.49 & $0.34 \$$ & $2.35^{*}$ \\
\hline Education & 0.08 & 0.76 & $0.26 \$$ & $2.52^{\star *}$ & $0.31 \$$ & $2.96^{\star \star}$ \\
\hline Parity & 0.21 & 0.44 & -0.49 & -1.01 & $-1.01 \$$ & $-2.09^{*}$ \\
\hline \multicolumn{7}{|l|}{ Characteristics of households } \\
\hline Family size & 0.13 & 1.44 & -0.02 & -0.18 & -0.11 & -1.16 \\
\hline SLI & 0.21 & 1.16 & 0.26 & 1.44 & 0.03 & 0.18 \\
\hline Socioeconomic status & -0.03 & -0.21 & -0.14 & -0.86 & -0.02 & -0.14 \\
\hline Per-capita income & -0.12 & 1.16 & -0.01 & -0.09 & -0.13 & -1.19 \\
\hline $\mathrm{R}^{2}$ & \multicolumn{2}{|c|}{0.26} & \multicolumn{2}{|c|}{0.24} & \multicolumn{2}{|c|}{0.17} \\
\hline Adjusted $\mathrm{R}^{2}$ & \multicolumn{2}{|c|}{0.19} & \multicolumn{2}{|c|}{0.17} & \multicolumn{2}{|c|}{0.10} \\
\hline
\end{tabular}

\$Significant coefficient; ${ }^{*}$ Significant at $\mathrm{p}<0.05 ;{ }^{* *}$ Significant at $\mathrm{p}<0.01 ;{ }^{* *}$ Significant at $\mathrm{p}<0.001$; LAZ=Length-for-age; SLI=Standard of living index; WAZ=Weight-for-age; WLZ=Weight-for-length

\begin{tabular}{|c|c|c|}
\hline \multirow{2}{*}{ Variable } & \multicolumn{2}{|c|}{$\begin{array}{c}\text { Complementary } \\
\text { feeding index }\end{array}$} \\
\hline & $\begin{array}{l}\text { Coef- } \\
\text { ficient }\end{array}$ & $t$-test \\
\hline \multicolumn{3}{|c|}{ Characteristics of children } \\
\hline Sex & 0.019 & 0.260 \\
\hline Age (months) & 0.26 & 1.47 \\
\hline Birth-order & $0.92^{s}$ & $2.04^{*}$ \\
\hline \multicolumn{3}{|c|}{ Characteristics of mothers } \\
\hline Age & -0.01 & -0.83 \\
\hline Education & 0.08 & 0.86 \\
\hline Parity & $-0.99^{\$}$ & $-2.15^{*}$ \\
\hline \multicolumn{3}{|c|}{ Characteristics of households } \\
\hline SLI & 0.01 & 0.09 \\
\hline Per-capita income & $0.22^{\mathrm{s}}$ & 2.201 \\
\hline Family size & 0.09 & 0.97 \\
\hline $\mathrm{R}^{2}$ & \multicolumn{2}{|c|}{0.28} \\
\hline Adjusted $\mathrm{R}^{2}$ & \multicolumn{2}{|c|}{0.22} \\
\hline
\end{tabular}

before nine months of age, which, in turn, can be attributed to delayed initiation of CF and the low food-frequency scores compared to the latter agegroup. In general, the CFI increased with age. The same has been reported by a study in slums and paediatric clinics of Gujarat, India (7). In multivariate analysis, a significant inverse association was observed between the age of the child and the nutritional status, which again indicates inadequacy in CF diets influencing the nutritional status of infants with increasing age.

Although a considerable proportion of the mothers reported feeding the recommended frequency of complementary foods (10) to their children, the low dietary diversity scores in both the agegroups suggest that food items from only 1-2 foodgroups were being fed to the infants throughout 6-12 months, thus indicating the monotony and lack of variety in their diets. It has been reported that diversity in the diet of child was significantly associated with the nutritional status, especially height-for-age index in sub-Saharan children (15). The present study also reports the same findings with dietary diversity for 9-12 months old infants being significantly associated with weight-for-age, 
length-for-age, and weight-for-length indices of nutritional status.

The food-frequency scores of CFI showed that cereals and diluted animal milk were the major foodgroups fed to the infants in this setting. The consumption of pulses in the sample was stumpy, with a very low frequency of feeding in the age-group of 6-8 months. The intake of meat was almost nil in the sample, and consumption of egg was also very less, especially among the age-group of 6-8 months. The reason for the low food-frequency scores for meat and egg was that infants were not fed meat and egg, thinking that they would not be able to digest them. Only a few mothers reported feeding egg-yolk mixed with hot milk to their infants during winter. Vegetables and fruits, though consumed by the other family members, were not part of the diet of infants. The low frequency of consumption of egg, meat, and poultry among infants and young children has also been previously reported in other countries (16-17).

Although bivariate analysis showed that maternal education was significantly associated with both CFI and nutritional status of infants, on multivariate analysis, maternal education was significantly $(\mathrm{p}<0.01)$ associated with nutritional status but not with CFI. Other studies have also reported that maternal education has a positive impact on the quality of care that she can provide to her offspring ( 5 , $6,12,16)$.

The impact of CFI on the nutritional status in the multivariate model in the study showed that, even after controlling for the maternal, child and household characteristics, the CF practices emerged as the important determinant of nutritional status. This finding contradicts the findings of a study in urban settings (7) where the composite index was unable to show an impact on nutritional status in the multivariate model. The CF practices which were identified to be undesirable in this sample were high use of baby-bottle, delayed initiation of $\mathrm{CF}$, and poor diversity of CFs fed to infants. Thus, the CFI developed reinforced what Arimond and Ruel had suggested earlier that feeding index like this can be used for identifying the specific CF practices that are deficient and also recognize vulnerable agegroups that need to be targeted through nutrition education and behaviour-change programme (7).

The findings of the present study showed an inverse significant relationship between parity of the mother with CFI and the nutritional status of infants. High parity would, in turn, leave less time for the mother for good infant-care and CF practices leading to poor nutritional status of the child.

The CFI was significantly associated with only length-for-age but not with weight-for-length and weight-for-age indices of the infants. Similar findings have been reported earlier (7). Ruel and Menon highlighted strong and significant associations between child-feeding practices and height-for-age index of the child, especially after 12 months of age, which the authors attributed to 'clustering of positive practices amongst older infants' that become apparent only after 12 months of age and increase over time (5). Contrary to these findings, Arimond and Ruel found that child-feeding index was not associated with height-for-age in rural African children (16).

The findings of the present study suggest the ability of CFI to reflect chronic malnutrition among infants. The CFI was used for reflecting the quality of diet of infants in terms of their food-frequency and dietary diversity scores. In the present study, by using the CFI, the poor quality of CFs being fed to infants was identified as a major lacuna determining the nutritional status of infants. Thus, this suggests an improvement in the dietary diversity and quality of complementary food as a major intervention for improving the CF practices and nutritional status of infants aged 6-12 months.

Since the CFI is a composite of key CF practices, in its present form, it can be used as an important tool in public-health programme for reflecting the CF practices as a whole, monitoring change, and also depicting the influence of critical CF practices on the nutritional status of infants.

Although the link between malnutrition and CF practices has been well-established (2), efforts to measure and quantify the CF practices have been limited till date due to methodological constraints. The CFI developed in the present study is an exploratory attempt to summarize and quantify the key CF practices into a composite index. This index would serve as an easy programme planning and monitoring tool by helping the programme planners to identify and target deficient CF practices and vulnerable groups for planning more effective nutrition education and behaviour-change communication and also monitor programme impact on improved CF practices.

\section{ACKNOWLEDGEMENTS}

The authors acknowledge the University Grants Commission, India, for providing financial support for the study. 


\section{REFERENCES}

1. World Health Organization. Physical status: the use and interpretation of anthropometry: report of WHO Expert Committee. Geneva: World Health Organization, 1995:161-262. (Technical report series no. 854).

2. Brown K, Dewey KG, Allen H. Complementary feeding of young children in developing countries: a review of current scientific knowledge. Geneva: World Health Organization, 1998:15-44. (WHO/NUT/98.1).

3. World Health Organization. Complementary feeding: report of global consultation, 10-13 December 2001 and summary of guiding principles. Geneva: World Health Organization, 2001:9-20.

4. International Institute for Population Sciences. National family health survey-3 (NFHS-3), 2005-2006, India. Mumbai: International Institute for Population Sciences, 2007:267-86.

5. Ruel MT, Menon P. Child feeding practices are associated with child nutritional status in Latin America: innovative uses of the demographic and health surveys. J Nutr 2002;132:1180-7.

6. Srivastava N, Sandhu A. Index for measuring child feeding practices. Indian J Pediatr 2007;74:363-8.

7. Arimond M, Ruel MT. Progress in developing infant and young child feeding index: an example from Ethopia demographic and health survey 2000. Washington, DC: Food and Nutrition Technical Assistance Project, Academy for Educational Development, 2002:4-13.

8. International Institute for Population Sciences. National family health survey-2, 1998-1999, India. Mumbai: International Institute for Population Sciences, 2000:251-73.

9. World Health Organization. Measuring changes in nutritional status: guidelines for assessing the nutri- tional impact of supplementary feeding programs for vulnerable groups. Geneva: World Health Organization, 1983:12-20.

10. Pan American Health Organization. Guiding principles for complementary feeding of breastfed child. Washington, DC: Pan American Health Organization, 2003:8-26.

11. World Health Organization. WHO child growth standards. Length-for-age, weight-for-age, weightfor-length, weight-for-height and body mass indexfor-age. Methods and Development. Department of Nutrition for Health and Development. Geneva: World Health Organization, 2006:50-221.

12. Amar-Klemesu M, Ruel MT, Maxwell DG, Levin CE, Morris SS. Poor maternal schooling is the main constraint to good child care practices in Accra. J Nutr 2000;130:1597-607.

13. Ghosh S, Kilaru A, Ganapathy S. Nutrition education and infant growth in rural Indian infants: narrowing the gender gap. JIMA 2002;100:1-10.

14. Vir SC. Nutritional status of children in Uttar Pradesh. Lucknow: Nutrition Foundation of India Archive, 2001:1-4.

15. Arimond M, Ruel MT. Dietary diversity is associated with child nutritional status: evidence from 11 demographic and health surveys. J Nutr 2004;134:257985.

16. Ntab B, Simondon KB, Milet J, Cissé B, Sokhna C, Boulanger $\mathrm{D}$ et al. A young child feeding index is not associated with either height-for-age or height velocity in rural Senegalese children. J Nutr 2005;135:45764.

17. Simondon KB, Simondon F. Infant feeding and nutritional status: the dilemma of mothers in rural Senegal. Eur J Clin Nutr 1995;49:179-88. 